\title{
Trialkyllead compounds induce the opening of the MTP pore in rat liver mitochondria
}

\author{
Marcantonio Bragadin ${ }^{\mathrm{a}, *}$, Daniele Marton ${ }^{\mathrm{b}}$, Sabrina Manente ${ }^{\mathrm{a}}$ \\ a Environmental Sciences Department, Ca' Foscari University of Venice, Dorsoduro D2137, 30123 Venice, Italy \\ ${ }^{\mathrm{b}}$ Inorganic, Metallorganic and Analytical Chemistry Department, Padua University, Via Marzolo 1, 35131 Padua, Italy
}

Received 12 September 2006; received in revised form 29 January 2007; accepted 29 January 2007

Available online 11 February 2007

\begin{abstract}
The interactions of the tributyl, triethyl and trimethyllead compounds with energized mitochondria have been investigated in this paper. It has been shown that the (alkyl $)_{3} \mathrm{~Pb}-\mathrm{Cl}$ compounds induce swelling in mitochondria suspended in a sucrose medium. The phenomenon is more marked the higher the lipophilicity and occurs in the following order: $(\mathrm{Bu})_{3} \mathrm{~Pb}>(\mathrm{Et})_{3} \mathrm{~Pb}>(\mathrm{Me})_{3} \mathrm{~Pb}$. As swelling is inhibited by cyclosporine, this suggests that the swelling is due to the opening of a trans-membrane pore (MTP pore) in the mitochondria. As this pore can be responsible for the inhibition of the ATP synthesis, and, consequently for cell death, the opening of the pore could be one of the reasons for the toxicity of the $(\text { alkyl })_{3} \mathrm{~Pb}-\mathrm{X}$ compounds.
\end{abstract}

(C) 2007 Elsevier Inc. All rights reserved.

Keywords: Mitochondria; Trialkyllead; Membrane channel

Lead compounds such as tetramethyl-lead and ethyllead have been widely utilized as antiknock compounds in fuels. Their presence in the environment gives rise to environmental problems, since the organolead compounds are very toxic, and the nervous system is the preferred target [1-3]. However, the persistence of these (alkyl) ${ }_{4} \mathrm{~Pb}$ compounds in the environment is not particularly high, since they are metabolized into the trialkyl (alkyl) ${ }_{3} \mathrm{~Pb}-\mathrm{X}$ compounds [4] and the toxicity is probably due to the presence of the latter $[4,5]$.

The toxicity of the trialkyllead compounds was studied "in vitro", in order to find the molecular, or biological target responsible for the "in vivo" effects. The mitochondria were studied in depth as, in many cases, they are responsible for acute toxic effects [6-10]. This is a consequence of the fact that the damage to the mitochondria, which produce energy for the cell (in the form of ATP) induces an equivalent cell damage. In the case of the (alkyl) ${ }_{3} \mathrm{~Pb}-\mathrm{X}$ compounds, many mechanisms which could induce ATP synthesis inhibition

\footnotetext{
${ }^{*}$ Corresponding author. Tel.: +39 041257 8507; fax: +39 0412578584.

E-mail address: bragadin@unive.it (M. Bragadin).
}

have been proposed. In particular, Aldridge et al. [7] proposed that the $(\mathrm{alkyl})_{3} \mathrm{~Pb}-\mathrm{X}$ compounds enter the mitochondria as (alkyl $)_{3} \mathrm{~Pb}-\mathrm{Cl}$ and are extruded as (alkyl) ${ }_{3} \mathrm{~Pb}-$ $\mathrm{OH}$, thus acting as $\mathrm{Cl}^{-} / \mathrm{OH}^{-}$ion exchangers. Alternatively, Bragadin et al. [6] proposed that the compounds enter the mitochondrial matrix as $(\text { alkyl })_{3} \mathrm{~Pb}^{+}$compounds and are extruded as (alkyl $)_{3} \mathrm{~Pb}-\mathrm{OH}$, thus giving rise to a cyclic mechanism which enhances the mitochondrial proton permeability. This does not exclude the fact that other mechanisms can induce an energy collapse in mitochondria (ATP synthesis inhibition).

The aim of this paper is to give evidence that (alkyl) ${ }_{3} \mathrm{~Pb}-\mathrm{X}$ compounds induce the opening of a trans-membrane pore, which inhibits ATP synthesis [11,12]. It should be noted that this is a "molecular", not a toxicological study. However, it will prove to be useful for a subsequent toxicological study, where the various molecular effects (mechanisms which induce ATP synthesis inhibition) are compared, in order to identify the effect which requires the lowest dose of toxic compound.

In mitochondria, the free energy arising from the oxidation of the substrates is stored and subsequently utilized, to 
synthesize the ATP. The oxidation occurs by means of a sequence of redox couples: i.e. the mitochondrial respiratory chain (RC). The electron flow in the RC is coupled with a stoichiometric proton extrusion and, since the mitochondrial membrane is not permeant to the protons, their extrusion gives rise to both a $\Delta \mathrm{pH}$ and a $\Delta \Psi$. The sum of these two terms

$\Delta \mathrm{pH}+\Delta \Psi=$ p.m.f.

is called the proton-motive force (p.m.f.) and in this form, the energy is stored and subsequently utilized to synthesize the ATP from the ADP [13]. The crucial factor concerning this mechanism is that the membrane is not permeant to the protons. Therefore, it follows that, any mechanism which induces an enhancement of the proton permeability, also induces ATP synthesis inhibition.

In recent years, it has been observed that many chemical compounds induce the opening of large size pores $[11,12]$ (MTP pores). The size of these pores allows for the passage of large molecules (molecular mass about $1500 \mathrm{Da}$ [11]) and consequently, of the protons. Therefore, the opening of an MTP pore implies an inhibition of the ATP synthesis. The opening of an MTP pore (which is inhibited by means of Cyclosporine [12]) is evidenced by means of swelling experiments: if the mitochondria are resuspended in a sucrose medium, the sucrose does not enter into the matrix, but, if the opening of the MTP pore is induced, the sucrose enters into the matrix. The entry of the sucrose is accompanied by the entrance of water and, as a consequence of a colloid osmotic effect, [14] a swelling of the mitochondria occurs [11-14]. The swelling can easily be monitored spectrophotometrically, since it induces an absorbance quenching at $540 \mathrm{~nm}$.

The absorbance quenching of the mitochondrial suspension induced by (methyl) ${ }_{3} \mathrm{~Pb}-\mathrm{Cl}$, (ethyl) ${ }_{3} \mathrm{~Pb}-\mathrm{Cl}$ and (butyl) $)_{3} \mathrm{~Pb}-\mathrm{Cl}$ is reported in Fig. 1. This effect is inhibited by means of Cyclosporine, thus indicating, as with the other organometallic compounds [15], that the swelling (which is correlated with the absorbance quenching) is due to the opening of the MTP pore (and not to other mechanisms such as the denaturants, which also allow for the passage of solutes, but the phenomenon is not inhibited by the presence of Cyclosporine [14]). The swelling depends on the lipophilicity of the compounds. This implies that the more lipophilic the compound, the lower the dose necessary to obtain the same effect. Therefore, the efficacy is in the following order:

butyl $>$ ethyl $>$ methyl.

For comparison purposes, the effect of phenylarsine oxide, a typical inducer of the opening of the MTP pore, is shown in Fig. 1d $[15,19]$.

The swelling of mitochondria, induced by the $(\text { alkyl })_{3} \mathrm{~Pb}-\mathrm{X}$ compounds has already been observed [7,8],

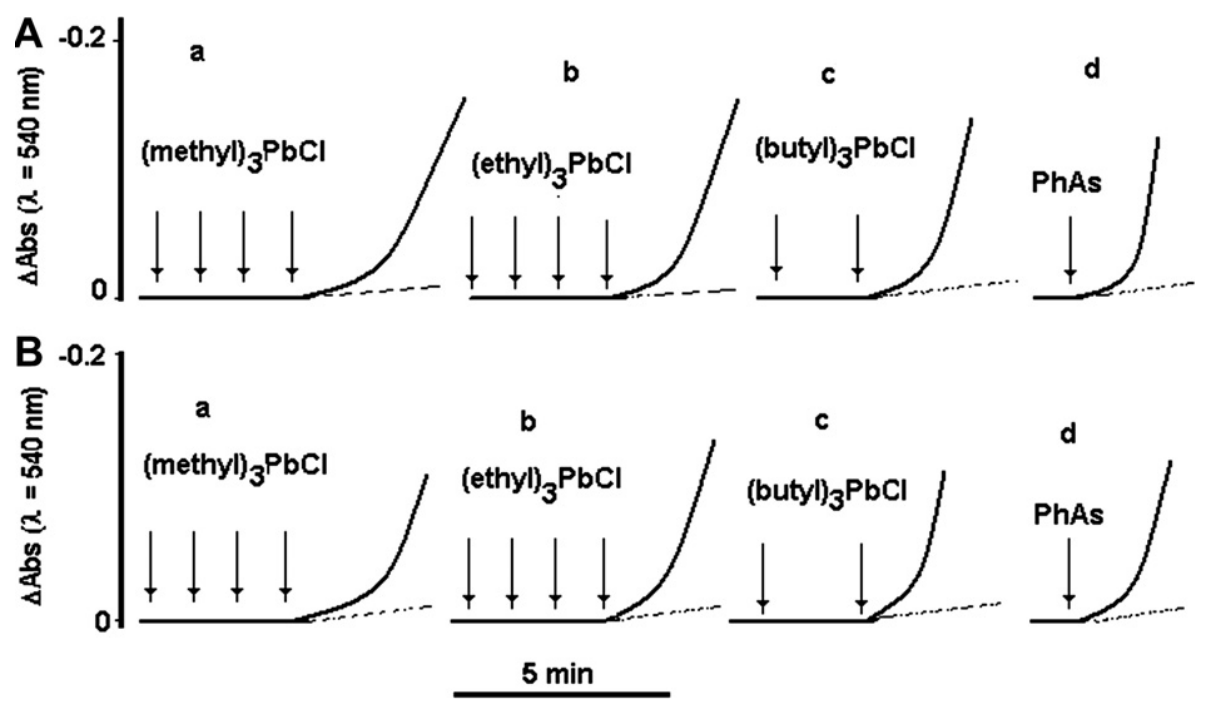

Fig. 1. Trialkyllead compounds induce the opening of the MTP pore. In A, medium composition (2.5 $\mathrm{ml}): 0.25 \mathrm{M}$ sucrose, $10 \mathrm{mM}$ Hepes-Mops pH 7.4, $1 \mathrm{mM} \mathrm{MgSO} 4,2 \mathrm{mM}$ sodium succinate, $0.5 \mathrm{mM}$ EGTA. The final concentration of mitochondria, final concentration: $0.5 \mathrm{mg} / \mathrm{ml}$. The protein concentration of the mitochondria was monitored by means of the Lowry procedure [17]. The mitochondria were prepared following the procedure indicated in Ref. [18]. After the addition of the mitochondria, the Jenway 6400 spectrophotometer was reset at zero absorbance, with an operative wavelength of $540 \mathrm{~nm}$. In this condition, the addition of trialkyllead compounds causes an Absorbance quenching, suggesting the opening of the MTP pore. Each arrow indicates in (a) the addition of $5 \mu \mathrm{M}$ (methyl) $)_{3} \mathrm{~Pb}-\mathrm{Cl}$, in (b), the addition of $2 \mu \mathrm{M}(\mathrm{ethyl})_{3} \mathrm{~Pb}-\mathrm{Cl}$, in (c), the addition of $1 \mu \mathrm{M}(\text { butyl })_{3} \mathrm{~Pb}-$ $\mathrm{Cl}$. It is evident that the same phenomenon is monitorable in (a) with a dose no less than $20 \mu \mathrm{M}$ (methyl) ${ }_{3} \mathrm{~Pb}$, in (b) no less than $8 \mu \mathrm{M}$ (ethyl) $)_{3} \mathrm{~Pb}$, in (c), no less than $2 \mu \mathrm{M}$ (butyl $)_{3} \mathrm{~Pb}$. Since the same behaviour appears in $\mathrm{B}(\mathrm{KCl}$ medium), this leads to the conclusion that the capacity to induce the opening of the pore follows the butyl $>$ ethyl $>$ methyl sequence. The dotted line indicates that the same experiment was performed in the presence of $1 \gamma$ Cyclosporine. The effect of the Cyclosporine confirms (and is the crucial proof) that the Absorbance quenching induced by the trialkyl lead compounds is due to the opening of the MTP pore. For comparison purposes, the effect of $20 \mu \mathrm{M}$ of phenylarsine oxide (PhAs), which is an inducer of the opening of the MTP pore, is shown in (d) [19]. Also in this case, it can be seen that the effect of the Cyclosporine (dotted line) is the inhibition of the opening of the pore (with Absorbance quenching). In B the conditions were the same, but the medium composition was different. Medium composition B: $0.125 \mathrm{M}$ KCl, $10 \mathrm{mM}$ Hepes-Mops pH 7.4, 1 mM $\mathrm{MgSO}_{4}, 2 \mathrm{mM}$ sodium succinate, $0.5 \mathrm{mM}$ EGTA. 
but the interpretation was different from ours. It was suggested that the swelling was due to the exchange between (alkyl $)_{3} \mathrm{~Pb}-\mathrm{OH}$ and $(\text { alkyl })_{3} \mathrm{~Pb}-\mathrm{Cl}$ in the mitochondrial matrix. However, at the time when this type of mechanism was presumed to be the correct one, the existence of a transmembrane pore (and its Cyclosporine sensitivity) had not yet been individualized.

Our interpretation is further supported by the fact that the concentrations of trialkyllead are too low to justify a swelling and, above all, by the fact that our medium contains sucrose (Fig. 1A), while chloride was absent (in the experiments of Fig. 1A) Both of these factors are not compatible with an $\mathrm{Cl}^{-} / \mathrm{OH}^{-}$ion exchange.

We have excluded that the observed swelling could be due to the concomitant presence of $\mathrm{Pb}^{2+}$ and $\mathrm{Ca}^{2+}[21]$ as contaminants or, in the case of $\mathrm{Pb}^{2+}$ that it was created by reduction of the $\mathrm{Pb}^{4+}$ of the alkylcompounds by means of the mitochondrial respiratory chain, since we operated in $\mathrm{Ca}^{2+}$ free medium. In addition, the results are the same if $0.5 \mathrm{mM}$ of EGTA (Fig. 1 legend) is present in the medium. It has been observed that EGTA reverses the possible effects of $\mathrm{Ca}^{2+}$ and/or $\mathrm{Pb}^{2+}$ as inducers of the opening of the pore [21].

In addition, the opening of the pore can explain certain phenomena observed in the cells, such as the $\mathrm{Ca}^{2+}$ release which occurs in the presence of micromolar concentrations of (alkyl) ${ }_{3} \mathrm{~Pb}-\mathrm{X}$ compounds [16].

Although this is not a toxicological work, it should be noted that the toxicity of the organometals in many, (but not all) whole organisms, generally follows the lipophilicity order as it did in our "in vitro" experiments [20]. This consideration, together with the most relevant data regarding the opening of the cyclosporine-sensitive pore (MTP), could offer an explanation for the molecular causes of this type of toxicity.

\section{References}

[1] C.B. Burns, B. Currie, New Zeal. J. Med. 25 (1995) 197-202.

[2] R.M. Booze, C.F. Mactutus, Experientia 46 (1990) 292-299.

[3] T. Nielsen, K.A. Jensen, P. Grandjeau, Nature 274 (1978) 602-604.

[4] J.E. Cremer, Br. J. Ind. Med. 16 (1959) 191-196.

[5] J.E. Cremer, S. Calloway, Br. J. Ind. Med. 18 (1961) 277-283.

[6] M. Bragadin, D. Marton, M. Murgia, V. Rizzoli, G. Scutari, R. Deana, J. Inorg. Biochem. 69 (1998) 259-262.

[7] W.N. Aldridge, B.W. Street, D. Skilleter, Biochem. J. 168 (1977) 353 364.

[8] W. Seinen, J.G. Vos, I. van Spanie, M. Snoek, R. Brands, H. Hooykaas, Toxicol. Appl. Pharm. 42 (1977) 197-212.

[9] W.N. Aldridge, J.E. Cremer, C.J. Therelfall, Biochem. Pharm. 11 (1962) 835-846.

[10] H. Kumulainen, S.C. Bondy, Toxicol. Appl. Pharm. 88 (1987) 77-86.

[11] P. Bernardi, V. Petronilli, J. Bioenerg. Biomembr. 28 (1996) 131-138.

[12] A. Nicolli, E. Basso, V. Petronilli, R.M. Wengers, P. Bernardi, J. Biol. Chem. 271 (1996) 2185-2192.

[13] P. Mitchell, Science 286 (1979) 1148-1159.

[14] P. Bernardi, Physiol. Rev. 79 (1999) 1127-1155.

[15] M. Bragadin, D. Marton, S. Manente, M. Grasso, A. Toninello, J. Inorg. Biochem. 89 (2002) 159-162.

[16] H. Kumulainen, S.C. Bondy, Toxicol. Appl. Pharmacol. 88 (1987) $77-86$.

[17] O.H. Lowry, N.J. Rosenbrough, D.L. Farr, R.J. Randall, J. Biol. Chem. 193 (1951) 265-271.

[18] M. Bragadin, T. Pozzan, G.F. Azzone, Biochemistry 18 (1979) 5972 5978.

[19] E. Lenartowicz, P. Bernardi, G.F. Azzone, J. Bioenerg. Biomembr. 23 (1991) 679-688.

[20] P.C. Graig, G. Eng, R.O. Jenkins, in: P.J. Craig (Ed.), Organometallic Compounds in the Environment, John Wiley and Sons Ltd., 2003.

[21] L. He, A.T. Poblenz, C. Medrano, D.A. Fox, J. Biol. Chem. 275 (2000) 12175-12184. 